\title{
Man ist sich des Wurzelgeflechts ihrer unzähligen Anwendungen in allen Wissenschaften wenig bewusst
}

\author{
Friedrich Götze im Gespräch
}

Lieber Herr Götze, Sie waren zwei Jahre lang Präsident der DMV und vorher zwei Jahre Vizepräsident. Was ist Ihre freudigste Erinnerung an diese Zeit?

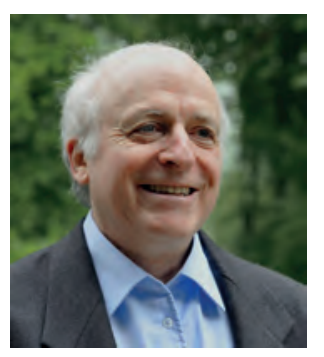

Ich habe mich 2018 als damaliger Vizepräsident sehr gefreut, dass sich nach mehreren überraschenden Absagen zur Jahrestagung der DMV 2020 eine engagierte Gruppe von Chemnitzer Kolleginnen und Kollegen unter Leitung von Peter Stollmann kurzfristig bereit erklärt hat, die Jahrestagung zu organisieren.

Die Tagung wurde angesichts der Pandemie nicht etwa verschoben, sondern den Organisatoren gelang es, eine Hybrid-Tagung mit überraschend starker, insbesondere auch internationaler Beteiligung sowie zahlreichen Minisymposien zu gestalten. Diese Tagung gibt Hoffnung für die weitere Entwicklung dieses für die Identität der DMV so wichtigen jährlichen Treffens.

Was ist die Aufgabe der Präsidentinnen und Präsidenten der DMV?

Sie sollten, unterstützt durch Vorstand und Präsidium, die Belange aller Mathematiker in der Universität, der Schule und der Industrie vertreten.

Weiterhin sind sie Ansprechpartner der Politik in Kooperation und mit Mandat aller Mathematik-Gesellschaften, um die Rolle der Mathematik in der zukünftigen Entwicklung von Ausbildung und technologischem Fortschritt zu verdeutlichen.

Wie sieht in Ihrer Wahrnehmung die Gesellschaft die Rolle der Mathematik?

Wahrscheinlich sieht man sie immer noch sehr beschränkt als ein Fach mit seinen über Jahrhunderte hochspezialisierten Theorie-Gebäuden und ist sich des Wurzelgeflechts ihrer unzähligen Anwendungen in allen Wissenschaften - nicht nur den MINT Bereichen - als einer fundamentalen Querschnitt- und Grundlagendisziplin wenig bewusst.
So wie ich selbst und auch andere erlebt haben, mit denen ich gesprochen habe, besitzen Sie eine besondere Begabung, als Botschafter der Mathematik gegenüber Politik und Gesellschaft zu wirken. Welche Botschaft liegt Ihnen besonders am Herzen?

Mir war immer sehr wichtig zu vermitteln, dass es die Ausbildung im mathematischen Denken und in mathematischen Begriffen ist, welche den intellektuellen Rahmen für konkrete Techniken und Verfahren in der Technik und bei der digitalen Transformation unserer Lebenswelt liefert. Dies trifft insbesondere für alle Bereiche der Informationswissenschaften zu, zum Beispiel KI, Datenanalyse und maschinelles Lernen. Leider werden diese zu häufig als von der Mathematik unabhängig gesehen.

Das ist eine tiefliegende und weitsichtige Botschaft. Wirtschaft und Politik haben es immer gerne sehr konkret und oft lieber kurzfristig sichtbar.

Ganz konkret und dringend ist, dass die Beherrschung grundlegender elementarer mathematischer Begriffe und Fertigkeiten für fast die Hälfte aller Studentinnen und Studenten eine notwendige Grundlage für ihr Studium ist - und natürlich auch dafür, dass sie sich überhaupt für MINT-Fächer in der Schule und im Studium begeistern können. Dieses für unsere Zukunft so wichtige Potential von Nachwuchstalenten darf insbesondere unter den Bedingungen der Pandemie nicht austrocknen, sondern sollte vergrößert werden.

Was waren Ihre zentralen Ziele und Schwerpunkte als Sie das Amt übernommen haben?

Ich denke, das wichtigste Thema war für mich der Übergang Schule-Hochschule. Dazu gab es gemeinsame Initiativen mit den großen naturwissenschaftlichen Gesellschaften und im Verbund mit den anderen mathematischen Fachgesellschaften. Da geht es konkret zum Beispiel um die Studierfähigkeit der Studienanfänger. Solche gemeinsamen Initiativen sind in meinen Augen auch deshalb sehr wichtig, weil sie die Gelegenheit bieten gemeinsam die Rolle der Wissenschaft in der Gesellschaft zu vermitteln. 
Wenn Sie zurückschauen, wo hätten Sie gerne mehr erreicht?

Ich hätte mir gewünscht, dass der von der DMV mit GDM und MNU initiierte Maßnahmenkatalog SchuleHochschule bei der KMK mehr bewirkt hätte. Allerdings lernt man in langjähriger Erfahrung im föderalen System Schule-Hochschule bescheiden zu sein. Ich hätte auch gerne die Fertigstellung einer Studie zur Bedeutung der Mathematik für die wirtschaftliche Zukunft eines Landes nach dem Vorbild solcher Studien in den Niederlanden und Großbritannien in meiner Amtszeit realisiert. Auch in der Einbindung jüngerer Kolleginnen und Kollegen in die Arbeit der DMV ist noch viel zu tun.

Was sehen Sie heute als Ihren wichtigsten Beitrag?

Ich bin sehr froh, dass mittlerweile die Interaktion mit allen mathematischen Gesellschaften besser funktioniert und die DMV in Absprache mit diesen die Vertretung der Mathematik mit Politik und im Verein aller naturwissenschaftlichen Fachgesellschaften übernimmt. Ferner bin ich froh, dass das wissenschaftliche Profil der DMV um die Hermann Minkowski-Medaille für herausragende Leistungen im mittleren Zeitabschnitt der akademischen Karriere ergänzt werden konnte.

Gerade in Ihrer Amtszeit gab es äußere Einflüsse, die es schwierig machten, Pläne zu verfolgen. Allen voran natürlich die Pandemie. Wie haben Sie das Heft des Handelns in der Hand behalten?

Im ersten Jahr war ich wie alle meine Vorgänger sehr viel unterwegs und habe zahlreiche Kontakte auf verschiedenen Ebenen und zu unterschiedlichen Anlässen pflegen können. Im Jahre 2020 wurde das durch die Absage fast aller Termine sehr viel schwieriger und die Fortsetzung der geplanten Vorhaben wurde dadurch behindert. Es gab allerdings auch überraschend erfreuliche Entwicklungen, wie die oben beschriebene Jahrestagung in Chemnitz.

Als Präsident müssen Sie vieles alleine stemmen. Dennoch habe ich Sie immer als einen überzeugten Teamplayer erlebt.

Ohne die Unterstützung durch Vorstand und Präsidium, die DMV-Beauftragten $z$. B. für die sehr erfolgreichen Gauß-Vorlesungen, die DMV-Medien und Netzwerkbüros (für Presse und Öffentlichkeitsarbeit), die DMVGeschäftsstelle sowie das Engagement der Organisatoren der Jahrestagungen und insbesondere des Herausgebers der Mitteilungen wäre dies nicht möglich gewesen.

Mein herzlicher Dank gilt auch den Mitgliedern und Unterstützern der DMV, insbesondere auch den lokalen Ansprechpartnern der DMV vor Ort an den Universitäten.

Sie haben das Amt an unsere neue Präsidentin Ilka Agricola übergeben. Was wünschen Sie ihr?

Ich wünsche Ihr und unserem neuen Vizepräsidenten Joachim Escher viel Erfolg bei ihrer Arbeit und hoffentlich bald wieder bessere Rahmenbedingungen für die Verwirklichung ihrer Ziele zur Entwicklung der DMV und der Mathematik in Deutschland.

Die Fragen stellte Sebastian Stiller. 